\title{
Experimental determination of base resistance contribution for point-like contacted c-Si solar cells using impedance spectroscopy analysis
}

\author{
A. Orpella*, I. Martín, J.M. López-González, P. Ortega, J. Muñoz, D.C. Sinde, \\ C. Voz, J. Puigdollers, R. Alcubilla \\ Departament d'Enginyeria Electrònica, Universitat Politècnica de Catalunya, C/ Jordi Girona 1- \\ 3, Mòdul C4, 08034 Barcelona, Spain.
}

${ }^{*}$ Corresponding author. Tel: +34 934017483, fax: +34 934016756, e-mail address: albert.orpella@upc.edu

Abstract: One of the most common strategies in high-efficiency crystalline silicon (c-Si) solar cells for the rear surface is the combination of a dielectric passivation with a point-like contact to the base. In such devices, the trade-off between surface passivation and ohmic losses determines the optimum distance between contacts or pitch. Given a certain pitch, the series resistance related to majority carrier flow through the base and the rear point-like contact ( $\left.R_{\text {base }}\right)$ is commonly calculated a-priori and not crosschecked in finished devices, since typical techniques to measure series resistance lead to an unique value that includes all ohmic losses. In this work, we present a novel method to measure $R_{\text {base }}$ using impedance spectroscopy (IS) analysis. The IS data at high frequencies allow to determine $R_{\text {base }}$ due to the presence of the capacitor formed by the metal/dielectric/semiconductor structure that covers most of the rear surface. The method is validated by device simulations where the dependence of $R_{\text {base }}$ on carrier injection, base resistivity and pitch are reproduced. Finally, $R_{\text {base }}$ is measured on finished devices. As a result, a more accurate value of the contacted area is deduced which is a valuable information for further device optimization.

Keywords: impedance spectroscopy, laser fired contacts, c-Si solar cells 


\section{Introduction}

High-efficiency c-Si solar cells demand excellent surface passivation with good carrier transport properties. At the rear surface of double-side contacted c-Si solar cells where the base contact is located, these requirements are often fulfilled by the combination of a dielectric passivating film with local contacts defined in a point-like pattern. This configuration was already applied in the high efficiency concepts developed in the 90's where the contacts were defined by photolithography [1-2]. In the last years, this idea has been revisited due to the introduction of laser techniques that allow the definition of the point-like pattern in a very cost-effective way. Particularly, three main strategies have been developed. Firstly, the laser beam is used to locally ablate the dielectric and the contacts are created in a subsequent full-area aluminum deposition and annealing [3-4]. A second approach consists of a metal deposited on top of the

dielectric that is laser fired through it leading to the so-called Laser Firing Contacts (LFC). Typically this technique is done with Aluminum to create base contacts on p-type substrates [5-6], but recently a metal stack containing Antimony has also demonstrated its viability for the creation of base contacts on n-type wafers [7]. Finally, the laser radiation can impinge directly on the dielectric film that works as dopant source. The advantage of this technique is that lower laser powers are required resulting in higher quality contacts. For $p$ type contacts, aluminum oxide [8] and boron doped silicon carbide films [9] have been reported, while for $n$-type contacts phosphorus doped silicon carbide has been applied [10-11]. 
When the base contact is defined in a point-like pattern, a trade-off between surface passivation and ohmic losses is found: the more contacted area fraction, the lower the ohmic losses, but the higher the surface recombination rate. Before device fabrication, an evaluation of this trade off to get the optimum contacted area fraction, i.e. optimum distance between contacts in a square matrix or pitch, is typically performed. Although $3 \mathrm{D}$ simulations lead to more accurate results [12-13], the most common approach is the reduction of the 3D problem to $1 \mathrm{D}$ by applying the equation proposed by Fischer [14], improved by Plagwitz and Brendel [15] and generalized by Saint-Cast et al. [16]. In this case, a theoretical value of the ohmic losses introduced by the point-like pattern can be calculated and expressed in a condensed parameter: the base resistance ( $\left.R_{\text {base }}\right)$. Typically, the nominal substrate resistivity and a contacted area depending on the technology are used as input parameters. This a-priori calculation could differ from the actual $R_{\text {base }}$ in the finished device. In addition, ohmic losses in the fabricated device can not be separated into different components when typical techniques to measure them are applied (see ref. [17] and references there in). However, an accurate knowledge of $R_{\text {base }}$ in finished solar cells is desirable for a reliable device development and, in particular, for pitch optimization.

In this work we present a novel method to determine $R_{\text {base }}$ in fabricated devices using impedance spectroscopy (IS) technique. This technique has been widely used to measure the frequency response of a large number of chemical systems, electronic materials and devices in several research areas such as biomedical or space applications [18-21]. In the photovoltaic field, it has been widely applied in dye-sensitized and organic solar cells [22-23], but also some 
studies have been also published on high-efficiency silicon solar cells revealing a trade-off between recombination and carrier transport in such devices [24-25]. Typically, IS technique allows to extract important parameters related to the physic structure of the solar cells - such as series and parallel resistances, carrier lifetime or diode ideality factor [26-28]. In this paper, we will focus on how the particular configuration of a dielectric passivated surface together with local base contacts impacts on IS measurements leading to the experimental determination of $R_{\mathrm{base}}$ in finished devices.

\section{2. $R_{\text {base }}$ measurement method validation}

\subsection{Model definition}

In the IS technique, the device is properly DC biased and a small AC signal is superposed to it. Typically, the measurement tool records the excitation AC voltage and the $\mathrm{AC}$ current response of the device resulting in a complex impedance $Z=Z^{\prime} \pm j Z^{\prime}$ for each applied frequency of the AC signal $(\omega)$. The obtained impedances are typically plotted in a $Z^{\prime},-Z^{\prime}$ " plane where the real part monotonically decreases with frequency. To validate the method to measure $R_{\text {base }}$ proposed hereby, we define a $n+/ p$ solar cell structure in ATLAS TCAD software with a blanket front emitter and a point-like rear contact following the same models that we used in ref. [29] where a detailed description of them can be found. The main characteristics of the solar cell are a substrate thickness $w$ of $260 \mu \mathrm{m}$, a nominal substrate resistivity $\rho_{\text {sub }}=1.8 \Omega \mathrm{cm}$ corresponding to $\mathrm{N}_{\mathrm{A}}=$ $7.8 \cdot 10^{15} \mathrm{~cm}^{-3}$, a $120 \Omega / \mathrm{sq}$ front $\mathrm{n}+$ emitter and the rear contact configuration where we define two alternatives (Fig. 1 sketches the simulated structures): 
- Point Contacted (PC): rear contact with a point-like square matrix with $600 \mu \mathrm{m}$ pitch and square contacts of $80 \times 80 \mu \mathrm{m}^{2}$. At these contacts, we define a surface recombination velocity $\left(S_{\text {cont }}\right)$ of $10^{6} \mathrm{~cm} / \mathrm{s}$ while for the passivated surface we use a surface recombination velocity $\left(S_{\text {pas }}\right)$ of 100 $\mathrm{cm} / \mathrm{s}$.

- Point Contacted and Metal Insulator Semiconductor structure (PC+MIS): both the geometrical and recombination parameters at the rear contact are the same than in PC structure, but in this case the dielectric film is included with the rear metal covering it. As dielectric film, we use a 100 nm-thick silicon oxide film with relative dielectric constant of 3.9. The metal was characterized by a metal workfunction equal to the c-Si bulk. With this condition and without any fixed charge density at the interface, the capacitance of this Metal Insulator Semiconductor (MIS) structure can be well approximated by the flat-band capacitance which is 26.75 $\mathrm{nF} / \mathrm{cm}^{2}$.

For these two structures, the IS measurements are simulated with frequencies ranging from $40 \mathrm{~Hz}$ to $45 \mathrm{MHz}$ for a bias voltage of $0.65 \mathrm{~V}$ and the obtained curves are plotted in Fig. 1. As it can be observed in Fig.1,(a), PC structure shows an unique semi-arc which is typically found in $\mathrm{p} / \mathrm{n}$ junctions and can be accurately modeled by solving the differential equation that controls carrier transport with a small signal bias. Assuming that the current through the $n+/ p$ junction is dominated by the electrons injected into the base, we can apply the expression for the IS response of well-passivated rear contacts that appears as the second term in equation (1) [30]. Apart from the small signal response of the $\mathrm{n}+/ \mathrm{p}$ junction, we add two lumped series resistances $R_{0}$ and $R_{\mathrm{base}}$. The former 
takes into account the ohmic losses related to the majority carrier flow through the $\mathrm{n}^{+}$emitter region and it is equal to $0.033 \Omega \mathrm{cm}^{2}$ determined from simulation results not shown in this work. The latter models the ohmic losses attributed to the majority carrier flow along the base which is closely related to the rear pitch and contact size. Notice that by fitting the IS response we can only obtain the sum of all the ohmic losses, since $R_{\text {base }}$ is connected in series to $R_{0}$ and there is no way to separate them from the IS data. However, the a-priori knowledge of $R_{0}$ will be useful to understand the physical origin of the IS model for PC+MIS structure introduced below. Combining the two series resistance with the $n+/ p$ junction response, we reach the following expression to fit the IS data:

$$
Z=R_{0}+\frac{R_{1} \operatorname{coth}\left(w / L \sqrt{1+j R_{1} C_{1} \omega}\right)}{\operatorname{coth}(w / L) \sqrt{1+j R_{1} C_{1} \omega}}+R_{\text {base }}
$$

where $w$ is the wafer thickness, $L$ is the effective diffusion length, $R_{1}$ is the small signal resistance of the $n+/ p$ junction and $C_{1}$ is the diffusion capacitance. These two parameters are typically used to determine the diode ideality factor and the minority carrier effective lifetime [28][31]. The simulated data can be excellently fitted by equation (1) using parameters shown in Table I. Very often, the accurate behavior of the $n+/ p$ junction is modeled by a lumped element circuit leading to the model shown in the inset of Fig. 1,(a) and IS response represented by the dashed line. This simplified model does not have $L$ as a parameter, which is responsible of the oval shape of the semi-arc, and is not able to fit the linear IS response at the $Z^{\prime},-Z^{\prime \prime}$ plane at high frequency known as 
Warburg effect. However, it matches the low frequency IS response and the Z'axis cross points which is enough to obtain the rest of the model parameters $R_{0}+R_{\text {base }}, R_{1}$ and $C_{1}$. In this case, $R_{1}$ can be identified as the width of the semiarc, $R_{0}+R_{\text {base }}$ corresponds to its shift from the $Z^{\prime}$-axis origin and $C_{1}$ is determined from the frequency at where the maximum $-Z$ " value is found. In other words, despite equation (1) excellently reproduces the simulated data with an assumable increase in complexity, the simple lumped model shown in figure 1 ,(a) is enough to determine $R_{1}, C_{1}$ and $R_{0}+R_{\text {base }}$ with similar accuracy.

In PC+MIS structure, the only difference to the previous structure is the presence of a MIS structure at the rear surface. As it can be seen in Fig. 1,(b), the semi-arc at high Z' values is identical to the previous one indicating that carriers follow the same electrical model than for PC structure with identical $L$, $R_{0}, R_{1}, C_{1}$ and $R_{\text {base }}$ values. This result is consistent with the fact that at these relatively low frequencies IS response is controlled by carrier recombination and this magnitude does not have changed between both structures. However, at high frequencies a second semi-arc arises indicating the appearance of a second capacitance $C_{2}$. The electrical model that explains this response is shown in the inset of Fig. 1,(b) and the physical explanation to it is the following. In this device, two carrier flows are superimposed. On the one hand, we have carriers that are related to device recombination and were already present on PC structure. These carriers are flowing through the point-like contact and are influenced by $R_{\text {base. }}$. On the other hand, there are carriers that flow through the $\mathrm{n}+\mathrm{p}$ junction and go to the MIS regions that cover the most part of the rear surface. These carriers travel vertically along the base and their ohmic losses are related to the substrate thickness and resistivity. Thus, a branch with $R_{2}$ and 
$C_{2}$ is connected in parallel to $R_{\text {base }}$ leading to the following expression to model the IS response (solid line in Fig. 1,(b)):

$$
Z=R_{0}+\frac{R_{1} \operatorname{coth}\left(w / L \sqrt{1+j R_{1} C_{1} \omega}\right)}{\operatorname{coth}(w / L) \sqrt{1+j R_{1} C_{1} \omega}}+\frac{R_{\text {base }} \cdot\left(j \omega C_{2} R_{2}+1\right)}{j \omega C_{2}\left(R_{\text {base }}+R_{2}\right)+1}
$$

As a first approach, the values for the new parameters can be calculated based on the device characteristics. $R_{2}$ can be expressed as $R_{2} \approx \rho \mathrm{w}$, where $\rho$ is the base resistivity. Although the nominal substrate resistivity $\left(\rho_{\text {sub }}\right)$ is a good approach for $\rho$, this value is modified by the carrier profile in the bulk that is strongly dependent on the bias voltage and the rear pitch. Regarding $C_{2}$, it is approximately the capacitance of the MIS structure at $0 \mathrm{~V}$ since the base is periodically contacted through the dielectric and, thus, it is at the same potential than the rear metal. Given the dielectric thickness and permittivity, the nominal capacitance at $0 \mathrm{~V}$ is a good approximation. However, this value is reduced by the total MIS area and by the fact that at the vicinity of the base contacts the capacitance is shortcircuited. As a result, the expressions mentioned above can be used as a first guess when the experimental data is to be fitted by the theoretical model. The values obtained from the best fit shown in Fig. 1,(b) agree well with this physical model (see Table I). $R_{2}$ is slightly lower than $\rho_{\text {sub }} w$ (a substrate resistivity of $1.4 \Omega \mathrm{cm}$ instead of the nominal substrate resistivity of $1.8 \Omega \mathrm{cm}$ can be deduced from this value related to minority carrier injection) while $C_{2}$ is $22 \mathrm{nF} / \mathrm{cm}^{2}$ which is very close to the $26.75 \mathrm{nF} / \mathrm{cm}^{2}$ deduced for the flat-band capacitance of the MIS structure. Notice that, as it was mentioned in the previous discussion, despite the low frequency semi-arc is not properly fitted by the lumped model shown in Fig. 1,(b) (dashed line), this model is accurate enough to fit the second semi-arc and determine $R_{\text {base }}$ which is the main goal of 
the work presented hereby. Additionally, the low $C_{2}$ value in the $\mathrm{nF} / \mathrm{cm}^{2}$ range results in a high impedance value at low frequencies. Consequently, the low frequency response is not impacted by the $R_{2}-C_{2}$ branch resulting in an identical semi-arc than for PC structure and a clear "visual" separation in the $Z,-Z$ ' plot of both behaviors.

As we have seen, the proposed model is able to explain the IS response of a solar cell whose rear surface is passivated with a dielectric film that does not have fixed charge density $\left(Q_{\mathrm{f}}\right)$ at the c-Si interface. However, typical films used for c-Si surface passivation, like silicon nitride or aluminum oxide, show nonnegligible $Q_{\mathrm{f}}$ values. In order to evaluate whether the proposed model is able to extract $R_{\text {base }}$ with a significant $Q_{\mathrm{f}}$ value, we repeat simulations of PC+MIS structure with $Q_{f}$ values of $10^{12}$ and $-10^{12} \mathrm{~cm}^{-2}$ at the rear c-Si/dielectric interface. The resulting IS responses (not shown in this work) are similar than for the case of $Q_{\mathrm{f}}=0 \mathrm{~cm}^{-2}$ with the high-frequency semi-arc starting at similar $Z^{\prime}$ coordinates. As a consequence, when the experimental data is fitted by the model presented hereby the resulting $R_{\text {base }}$ can be determined ( 0.273 and 0.275 $\Omega \mathrm{cm}^{2}$ for $Q_{\mathrm{f}}$ values of $-10^{12}$ and $10^{12} \mathrm{~cm}^{-2}$ respectively). This result is expected due to the high sheet resistance in the $k \Omega$ range of the inversion/accumulation layer. On the other hand, it must be mentioned that the $R_{2}$ and $C_{2}$ values determined under the presence of $Q_{\mathrm{f}}$ do not make physical sense $\left(R_{2}\right.$ higher than $\rho_{\mathrm{sub}} \cdot W=0.0468 \Omega \mathrm{cm}^{2}$ and $C_{2}$ equal for both cases with significant $Q_{\mathrm{f}}$ ) suggesting that the branch of the model that includes these elements used should be revised. A reasonable approach could be the replacement of $R_{2}$ and $C_{2}$ by a transmission line where every capacitance element has a series and shunt resistances associated with it due to the presence of the 
inversion/accumulation layer. The development of this model is beyond the scope of this paper and will be addressed in future works.

The physical understanding of the IS response of $\mathrm{PC}+\mathrm{MIS}$ structure is important; however, the most relevant result of this analysis is that the impact of $R_{\text {base }}$ can be now separated from the rest of the ohmic losses since it is linked to the second semi-arc (in broad terms it can be considered its width) instead of just a shift to higher Z' values. Taking advantage of it, we will be able to determine this parameter for a wide variety of conditions.

\subsection{Dependence of $R_{\mathrm{base}}$ on base resistivity}

We simulate the IS data of PC+MIS structure for bias voltages ranging from 0.6 to $0.7 \mathrm{~V}$. The evolution of the impedance data are plotted in Fig. 2. As it can be seen, the first semi-arc is strongly reduced when voltage increases due to the increase in the current flow through the device, i.e. the diode is forward biased. The second semi-arc shows a soft trend towards lower Z' values indicating that the associated series resistance reduces.

This trend is confirmed when we plot the $R_{\text {base }}$ values obtained from the best fit of the IS data with equation (2), as it is done in Fig. 3. In this figure, we also show the $R_{\text {base }}$ data calculated from the model proposed by Fischer for round shape contacts [14]:

$$
R_{\text {base }}=\rho \frac{p^{2}}{2 \pi r} \times \tan ^{-1}\left(\frac{2 w}{r}\right)+\rho w\left(1-e^{(-w / p)}\right)
$$


where $r$ is the contact radius, $p$ is the rear pitch and $w$ is the wafer thickness. The value chosen for the radius is $45.1 \mu \mathrm{m}$ leading to a round contact area equivalent to the square $80 \times 80 \mu \mathrm{m}^{2}$ contacts defined in the simulations. The calculated $R_{\text {base }}$ value applying equation (2) with $\rho=\rho_{\text {sub }}=1.8 \Omega \mathrm{cm}$ is shown in Fig. 3 with a dashed line. This value agrees well with the values deduced from IS data at low injection. Notice that $\rho$ is reduced as the voltage increases due to the injection of minority carriers. An estimation of the corresponding $\rho$ at every bias voltage $(V$ can be done assuming that the minority carrier density at the $\mathrm{c}$ Si bulk $(\Delta n)$ is equal to its value at the edge of the space charge region. This assumption introduces some error since the carrier distribution is not constant along the bulk; however, this error is minimized for a rear surface well passivated. Then, base resistivity can be calculated as follows:

$$
\rho=\frac{1}{q\left[\mu_{n} \Delta n+\mu_{p}\left(N_{A}+\Delta n\right)\right]}
$$

where $\Delta n$ is obtained applying the following expression:

$$
\Delta n=\frac{n_{i}^{2}}{N_{A}} \times e^{\left(q V_{j} / k_{B} T\right)}
$$

where symbols have their usual meanings and $V_{\mathrm{j}}$ is the voltage applied to the junction that can be calculated as $V_{\mathrm{j}}=V-J \times\left(R_{0}+R_{\text {base }}\right)$. The resulting $R_{\text {base }}$ values applying the expressions mentioned above (solid line in Fig. 3) reproduce the $R_{\text {base }}$ dependence. Another way to change base resistivity is varying the doping density. We simulate the IS data at $V=0.65 \mathrm{~V}$ for 4 and 0.8 $\Omega \mathrm{cm}$ substrate resistivities. The obtained $R_{\text {base }}$ values are also plotted in Fig. 3 with the corresponding calculation from the analytical model. All the extracted 
$R_{\text {base }}$ values agree well with the calculated ones demonstrating that the method to determine $R_{\text {base }}$ by IS data is reliable.

\subsection{Dependence of $\boldsymbol{R}_{\mathrm{base}}$ on pitch}

The ohmic losses introduced by a point-like contacted rear surface are strongly related to the rear pitch. We simulate the IS data at $\mathrm{V}=0.65 \mathrm{~V}$ for $\mathrm{PC}+\mathrm{MIS}$ structures with pitches ranging from 300 to $1600 \mu \mathrm{m}$ and we extract the $R_{\text {base }}$ values by fitting the data with the proposed model. In addition, we calculate the

corresponding $R_{\text {base }}$ from equation (2) using $\rho=\rho_{\text {sub }}=1.8 \Omega \mathrm{cm}$ and plot them in Fig. 4 (dashed line). As it can be seen, the analytical model follows the same trend than the data extracted from the simulations, but it diverges for longer pitches. In the long pitch range, surface passivation is improved and the excess carrier density that can be maintained at a certain voltage increases. Thus, the effect of base resistivity reduction by carrier injection is stronger. If we apply the calculations mentioned above to estimate $\Delta n$ and modify base resistivity, the analytical model (solid line in Fig. 4) agrees well with the simulated data demonstrating again the validity of the method proposed hereby.

\section{Results on experimental samples}

Now, we apply the method to determine $R_{\text {base }}$ in experimental samples. Particularly, high-efficiency crystalline silicon Passivated Emitter Rear Locally Contacted (PERC) solar cells have been used. These solar cells were fabricated using $1.8 \Omega \mathrm{cm}$ p-type $<100>\mathrm{FZ} \mathrm{c}$-Si 4" wafers with a thickness of $260 \mu \mathrm{m}$. Fabrication process is explained in detail in ref. [32], but in broad terms it can be summarized as follows. After front surface texturization, an homogeneous $\mathrm{n}^{+}$emitter was created by phosphorous diffusion with sheet 
resistance of about $100 \Omega / \mathrm{sq}$. Front and rear surfaces were passivated by thermally grown $\mathrm{SiO}_{2}$. A front contact grid based on $\mathrm{Ti} / \mathrm{Pd} / \mathrm{Ag}$ e-beam evaporation with $3 \%$ contacted area was defined, while the rear surface was covered by evaporated Aluminum and a LFC process was performed to create local rear contacts using IR (1064 nm) Nd:YAG pulsed laser. This LFC process leads to round laser spots on the Aluminum film with a radius of $40 \mu \mathrm{m}$. In Fig. 5 we show a sketch of the fabricated solar cell with the PC+MIS model superimposed in order to connect structural layers of the cell to the circuit element response.

In this work, we report the results for the $600 \mu \mathrm{m}$ pitch sample. We used impedance analyzer HP4294A to measure the IS response of this device from $40 \mathrm{~Hz}$ to $15 \mathrm{MHz}$ varying the bias voltage from $0.55 \mathrm{~V}$ to $0.74 \mathrm{~V}$. In order to get a lineal response from the solar cell, $\mathrm{AC}$ signal was fixed to $10 \mathrm{mV}$, lower than the thermal voltage at $25^{\circ} \mathrm{C}$. It must be mentioned that the solar cell was measured using a Printed Circuit Board (PCB) to directly contact it with a coaxial connection avoiding non-shielded wires. As a result of this configuration, the parallel parasitic capacitance and the series parasitic inductance have decreased to negligible values allowing a non-distorted measurement up to 15 $\mathrm{MHz}$. This set-up is of crucial importance in order to reduce parasitic elements that hinder the solar cell response at high frequencies, where the region of interest to determine $R_{\text {base }}$ is located.

In Fig. 6, we show the IS data for bias voltages ranging from 0.55 to $0.7 \mathrm{~V}$. The experimental data follow the same trend than the one observed for the simulated PC-MIS structure shown in Fig. 2. Moreover, the model proposed in 
this work is able to closely reproduce the experimental data (solid lines). Focusing on $R_{0}$ and $R_{\text {base }}$ parameters, which are the relevant ones in this work, best fits are obtained with a constant value for $R_{0}$ of $0.350 \Omega \mathrm{cm}^{2}$ (attributed to metal grid and contact resistances) while $R_{\text {base }}$ decreases with voltage as shown in Fig. 7. Again, this trend can be analytically modeled including carrier injection with the expressions mentioned above. However, the contact radius that leads to a best fit of the experimental data is $33 \mu \mathrm{m}$ in contrast to the $40 \mu \mathrm{m}$ deduced from the observation of the laser spot, indicating that the electrical contact is not created in the whole laser processed region. Consequently, $R_{\text {base }}$ at low injection is $0.490 \Omega \mathrm{cm}^{2}$ instead of $0.407 \Omega \mathrm{cm}^{2}$ that would be usually deduced from equation (3) with $40 \mu \mathrm{m}$ radius. The proposed method for $R_{\text {base }}$ determination is particularly well suitable for rear contact techniques where the real electrical contact size is not accurately known, as in laser fired contacts. In fact, recently a micro-scale study of the doping density distribution along the laser processed spots has been reported with evidences of low doped regions in the spot perimeter [33]. All this information is very important for an accurate development of these devices, in particular for the optimum pitch determination, and illustrates the usefulness of the method proposed in this work.

\section{Conclusions}

We have developed a novel method for $R_{\text {base }}$ determination in finished solar cells whose base contact is defined in a point-like structure at the rear surface. It is based on fitting IS measurements by a physical model that is able to reproduce the dependence of $R_{\text {base }}$ on carrier injection, substrate doping density and pitch, as it is demonstrated by device simulations. This method is applied to 
experimental solar cells whose p-type base is contacted by locally laser firing aluminum film. The experimental $R_{\text {base }}$ data show a decrease with bias voltage attributed to carrier injection and they are best fitted by an analytical model where the contact radius is $33 \mu \mathrm{m}$ instead of $40 \mu \mathrm{m}$ determined by laser spot observation. This information is crucial for further optimization of these devices, particularly optimum pitch determination.

\section{Acknowledgements}

This work has been funded by Spanish government through project TEC201126329 and it has also received funding from the European Union's Seventh Framework Programme for research, technological development and demonstration under grant agreement no. 608498 .

\section{References}

[1] J. Zhao, A. Wang, P.P. Altermatt, S.R. Wenham, M.A. Green, 24\% Efficient perl silicon solar cell: Recent improvements in high efficiency silicon cell research, Solar Energy Materials and Solar Cells 41 (1996) 87-99.

[2] M.A. Green, J. Zhao, A. Wang, S.R. Wenham, Very high efficiency silicon solar cells- Science and technology, IEEE Transactions on Electron Devices 46 (10) (1999) 1940-1947.

[3] W. Pfleging, A. Ludwig, K. Seemann, R. Preu, H. Mäckel and S. W. Glunz, Laser micromachining for applications in thin film technology, Applied Surface Science 154-155 (2000) 633-639. 
[4] P. Engelhart, S. Hermann, T. Neubert, H. Plagwitz, R. Grischke, R. Meyer, U. Klug, A. Schoonderbeek, U. Stute, R. Brendel, Laser Ablation of $\mathrm{SiO}_{2}$ for Locally Contacted Si Solar Cells With Ultra-short Pulses, Progress in Photovoltaics: Research and Applications 15 (2007) 521-527.

[5] E. Scheneidorlöchner, R. Preu, R. Lüdemann, W. Glunz, Laser-fired rear contacts for crystalline silicon solar cells, Progress in Photovoltaics: Research and Applications 10 (2002) 29-34.

[6] P. Ortega, A. Orpella, I. Martín, M. Colina, G. Lopez, C. Voz, M.I. Sánchez, C. Molpeceres, R. Alcubilla, Laser-fired contact optimization in c-Si solar cells, Progress in Photovoltaics: Research and Applications 20 (2012) 173-180.

[7] J. He, S. Hegedus, U. Das, Z. Shu, M. Bennett, L. Zhang, R. Birkmire, Laserfired contact for $\mathrm{n}$-type crystalline Si solar cells, Progress in Photovoltaics: Research and Applications (2014), http://dx.doi.org/10.1002/pip.2520, in press.

[8] P. Ortega, I. Martín, G. Lopez, M. Colina, A. Orpella, C. Voz, R. Alcubilla, Ptype c-Si solar cells based on rear side laser processing of $\mathrm{Al}_{2} \mathrm{O}_{3} / \mathrm{SiC}_{\mathrm{x}}$ stacks, Solar Energy Materials and Solar Cells 106 (2012) 80-83.

[9] B. Steinhauser, U. Jäger, J. Benick, M. Hermle, PassDop rear side passivation based on $\mathrm{Al}_{2} \mathrm{O}_{3} / a-\mathrm{SiC}_{x}: \mathrm{B}$ stacks for $\mathrm{p}$-type $\mathrm{PERL}$ solar cells, Solar Energy Materials and Solar Cells 131 (2014) 129.

[10] U. Jäger, D. Suwito, J. Benick, S. Janz, R. Preu, A laser based process for the formation of a local back surface field for n-type silicon solar cells, Thin Solid Films 519 (2011) 3827-3830. 
[11] I. Martín, M. Colina, A. Orpella, C. Voz, S. De Vecchi, T. DEsrues, S. Abolmasov, P. Roca i Cabarrocas, R. Alcubilla, Low recombination $n+$ regions created by $\mathrm{n}^{+} \mathrm{c}-\mathrm{Si}$ epitaxial layers and laser processing of phosphorus-doped SiCx films, in: Proceedings of $27^{\text {th }}$ European Photovoltaic Solar Energy Conference and Exhibition (2014) 1519-1523.

[12] K. Kotsovos, K. Misiakos, Three-dimensional simulation of carrier transport effects in the base of rear point contact silicon solar cells, Journal of Applied Physics 89 (2001) 2491-2496.

[13] M. Zanuccoli, R. De Rose, P. Magnone, E. Sangiorgi, C. Fiegna, Performance analysis of rear point contact solar cells by three-dimensional numerical simulation, IEEE Transactions on Electron Devices 59 (2012) 13111319.

[14] B. Fischer, Loss analysis of crystalline silicon cells using photoconductance and quantum efficiency measurements, PhD Tesis, University of Konstanz, 2003.

[15] H. Plagwitz, R. Brendel, Analytical model for the diode saturation current of point-contacted solar cells, Progress in Photovoltaics: Research and Applications 14 (2006) 1-12.

[16] P. Saint-Cast, M. Rüdiger, A. Wolf, M.Hofmann, J. Rentsch, R. Preu, Advanced analytical model for the effective recombination velocity of locally contacted surfaces, Jorunal of Applied Physics 108 (2010) 013705. 
[17] K.C. Fong, K. R. Mclntosh, A. W. Blakers, Accurate series resistance measurements of solar cells, Progress in Photovoltaics: Research and Applications 21 (2013) 490-499.

[18] E. Barsoukov, J.R. Macdonald, Impedance spectroscopy: theory, experiment, and applications, Wiley-Interscience, John Wiley \& Sons, Inc., 2005.

[19] U.G. Kyle, I. Bosaeus, A.D. De Lorenzo, P. Deurenberg, M. Elia, J.M. Gomez, B.L. Heitmann, L. Kent-Smith, J.-C. Melchior, M. Pirlich, H. Scharfetter, A.M.W.J. Schols, C. Pichard, Bioelectrical impedance analysis - part I: review of principles and methods, Clinical Nutrition 23 (2004) 1226-1243.

[20] M. S. Suresh, Measurement of solar cell parameters using impedance spectroscopy, Solar Energy Materials and Solar Cells 43 (1996) 21-28.

[21] R. Anil Kumar, M. S. Suresh, J. Nagaraju, Measurement and comparison of AC Parameters of silicon (BSR and BSFR) and gallium arsenide (GaAs/Ge) solar cells used in space applications, Solar Energy Materials and Solar Cells $60(2000)$ 155-166.

[22] F. Fabregat-Santiago, J. Bisquert, G.García-Belmonte, G. Boschloo, A. Hagfeldt, Influence of electrolyte in transport and recombination in dyesensitized solar cells studied by impedance spectroscopy, Solar Energy Materials and Solar Cells 87 (2005) 117-131. 
[23] F. Fabregat-Santiago, G. García-Belmonte, I. Mora-Seró, J.Bisquert, Characterization of nanostructured hybrid and organic solar cells by impedance spectroscopy, Physical Chemistry Chemical Physics 13 (2011) 9083-9118.

[24] I. Mora-Sero, G. García-Belmonte, P. P. Boix, M.A. Vazquez, J. Bisquert, Impedance spectroscopy characterisation of highly efficient silicon solar cells under different light illumination intensities, Energy Environmental Science 2 (2009) 678-686.

[25] G. García-Belmonte, J. García-Cañadas, I. Mora-Seró, J. Bisquert, C. Voz, J. Puigdollers, R. Alcubilla, Effect of buffer layer on minority carrier lifetime and series resistance of bifacial heterojunction silicon solar cells analyzed by impedance spectroscopy, Thin Solid Films 514 (2006) 254-257.

[26] D.J. Crain, J.E. Garland, S.E. Rock, D. Roy, Quantitative characterization of silicon solar cells in the electro-analytical approach: Combined measurements of temperature and voltage dependent electrical parameters, Analytical Methods 4 (2012) 106-117.

[27] D.J. Crain, S.E. Rock, J.E. Garland, D. Roy, Comparison of D.C. and A.C. electro-analytical methods for measuring diode ideality factors and series resistances of silicon solar cells, Current Applied Physics 13 (2013) 2087-2097.

[28] S. Kumar, Vandana, C.M.S. Rauthan, V.K. Kaul, S.N. Singh, P.K. Singh, Spectral and injection level dependence of recombination lifetimes in silicon measured by impedance spectroscopy, IEEE Journal of Photovoltaics 4 (2014) 380-386. 
[29] J.M. López-González, I. Martín, P. Ortega, A. Orpella, R. Alcubilla, Numerical simulations of rear point-contacted solar cells on $2.2 \Omega \mathrm{cm}$ p-type c-Si substrates, Progress in Photovoltaics: Research and Applications 23 (2014) 6977.

[30] J. Bisquert, G. García-Belmonte, F. Fabregat-Santiago, P.R. Bueno, Theoretical models for ac impedance of finite diffusion layers exhibiting low frequency dispersion, Jornal of Electroanalytical Chemistry 475 (1999) 152-163.

[31] I. Mora-Seró, Y. Luo, G. García-Belmonte, J. Bisquert, D. Muñoz, C. Voz, J. Puigdollers, R. Alcubilla, Recombination rates in heterojunction silicon solar cells analyzed by impedance spectroscopy at forward bias and under illumination, Solar Energy Materials and Solar Cells 92 (2008) pp. 505-509.

[32] P.Ortega, G. López, A. Orpella, I. Martín, M. Colina, C. Voz, S. Bermejo, J. Puigdollers, M. García, R. Alcubilla, Crystalline silicon solar cells beyond 20\% efficiency, in: Proceedings of $8^{\text {th }}$ IEEE Spanish Conference on Electron Devices (2011) 5744231.

[33] A. Roigé, J. Alvarez, J.P. Kleider, I. Martín, R. Alcubilla, L.F. Vega. Microscale Spatially Resolved Characterization of Highly Doped Regions in Laser-Fired Contacts for High-Efficiency Crystalline Si Solar Cells. Accepted in IEEE Journal of Photovoltaics. DOI: 10.1109/JPHOTOV.2015.2392945. 
Figure Captions

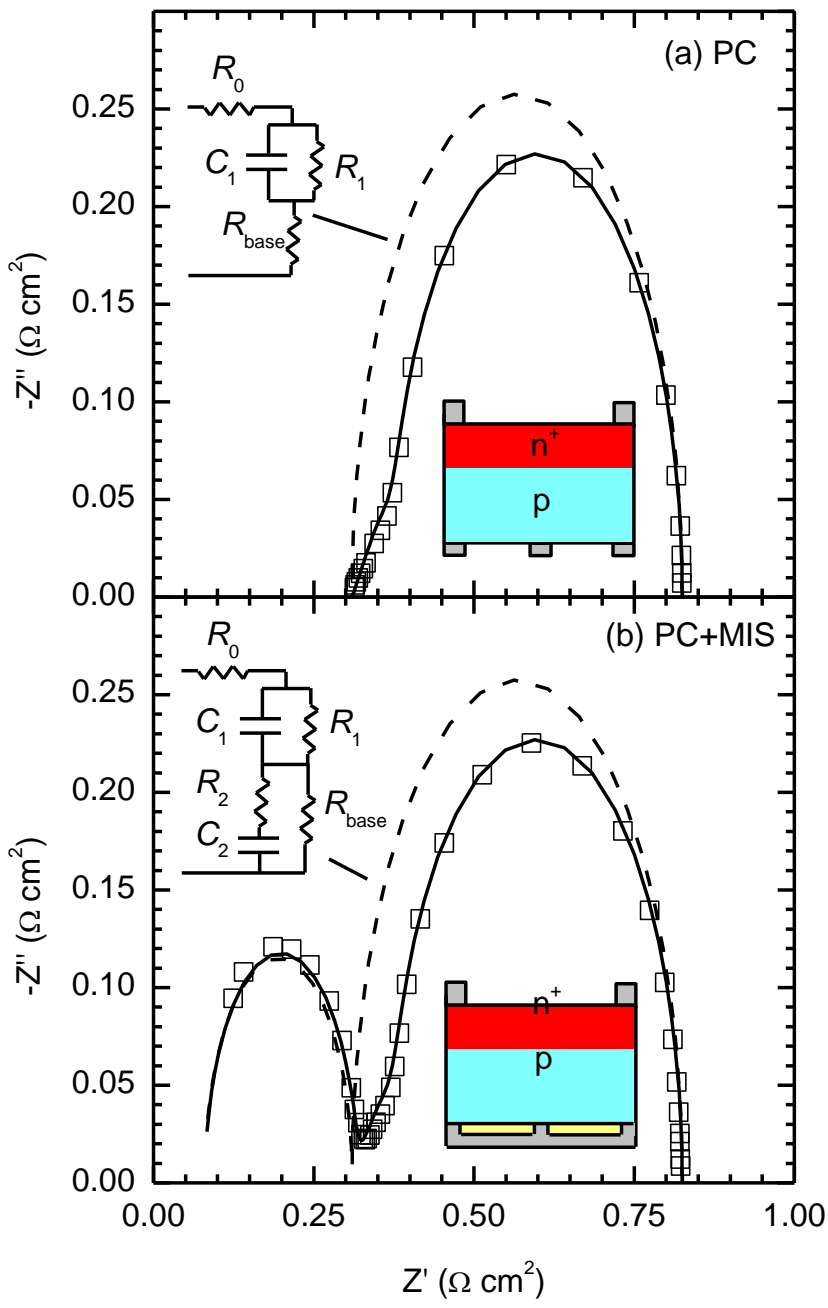

Fig. 1. Simulated IS data (symbols) and theoretical curves (solid lines) with parameters shown in Table 1 for (a) PC structure and (b) PC+MIS structure. Calculated curves using the simple lumped element model (dashed lines) with the same parameters are also shown. 


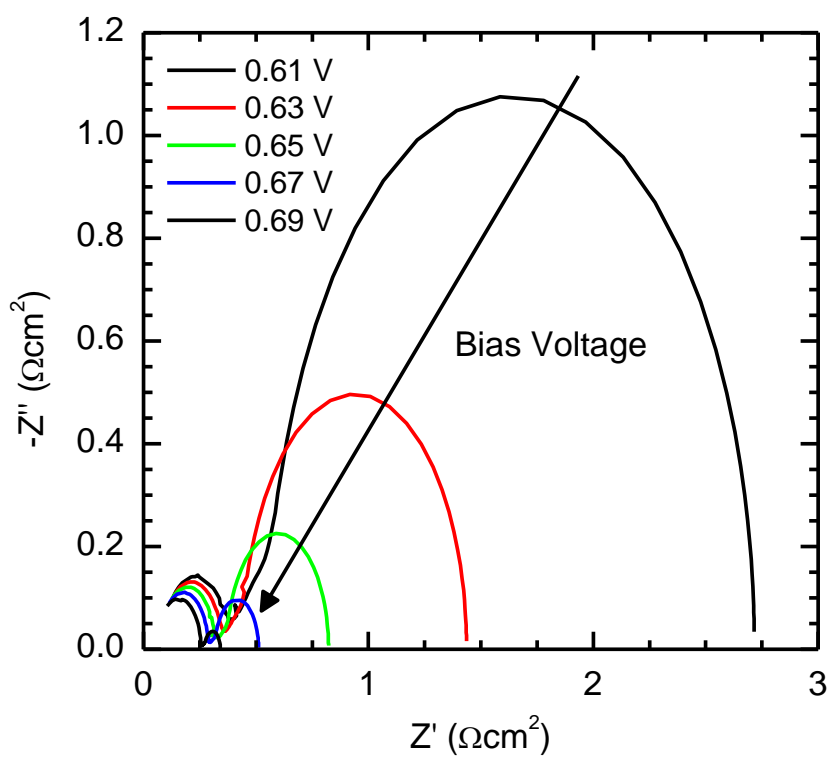

Fig. 2. Evolution of simulated IS data for PC+MIS structure at different bias voltages.

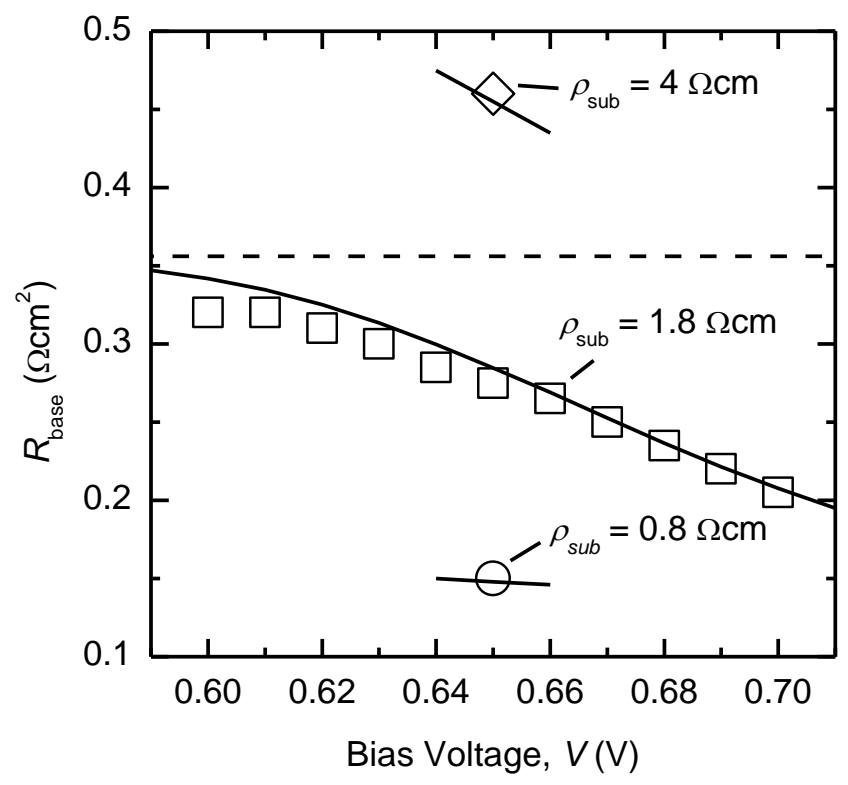

Fig. 3. $R_{\text {base }}$ vs. bias voltage (symbols) determined from the simulated IS data by the method proposed hereby. Theoretical curve (solid line) from the analytical model is able to reproduce the dependence of $R_{\text {base }}$ on carrier injection. The $R_{\text {base }}$ value using the nominal substrate resistivity (dashed line) is also shown. Additionally, $R_{\text {base }}$ values at $0.65 \mathrm{~V}$ for substrates resistivities of 4 and $0.8 \Omega \mathrm{cm}$ are also plotted and agree with the model. 


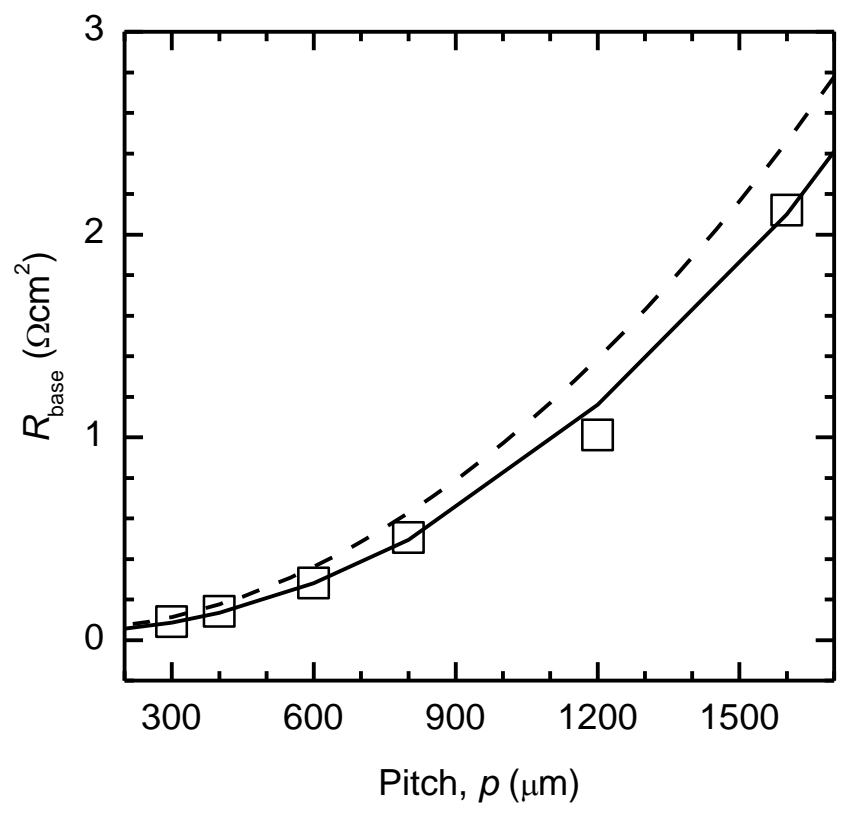

Fig. 4. Dependence of $R_{\text {base }}$ on pitch determined from simulated IS data for $\mathrm{PC}+\mathrm{MIS}$ structures at $0.65 \mathrm{~V}$. The predicted dependence using equation (3) with nominal substrate resistivity (dashed line) diverges for long pitches, whereas a good agreement is found when carrier injection is included (solid line).

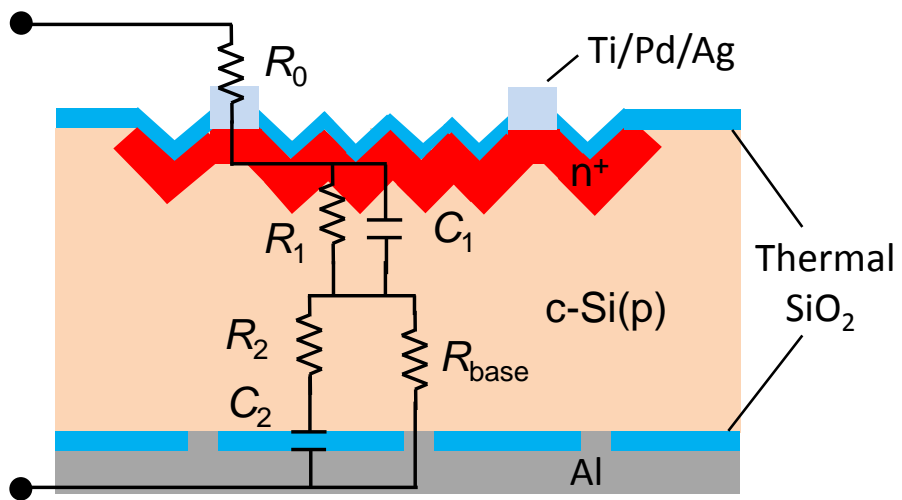

Fig. 5. Sketch of the fabricated solar cells with the connection between the different elements of the PC+MIS model to the physical layers of the device. 


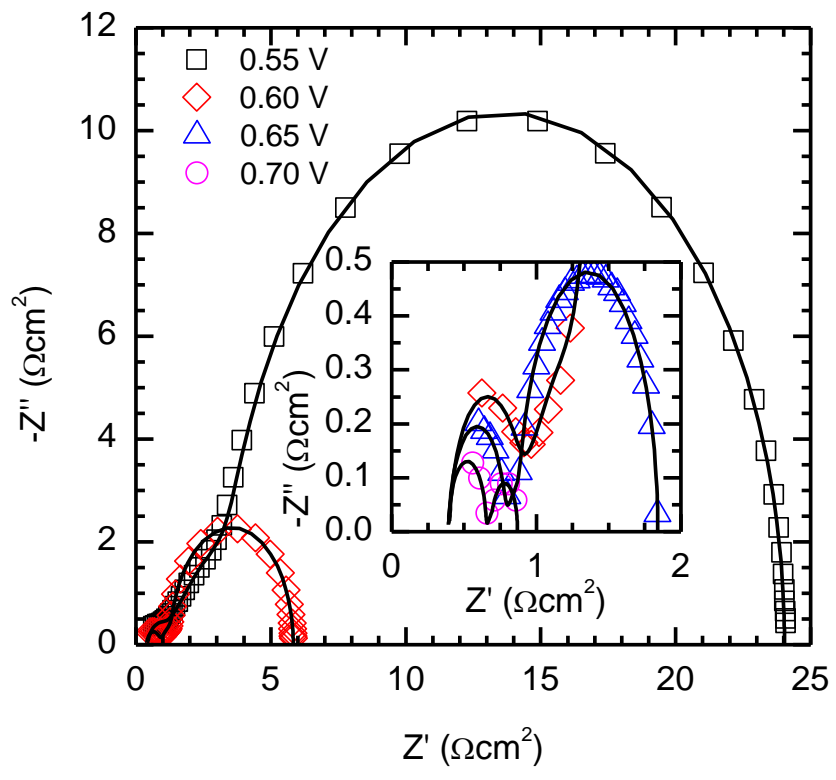

Fig. 6. Experimental IS data (symbols) and best fit with theoretical model (lines) for finished devices at voltages ranging from 0.55 to $0.7 \mathrm{~V}$.

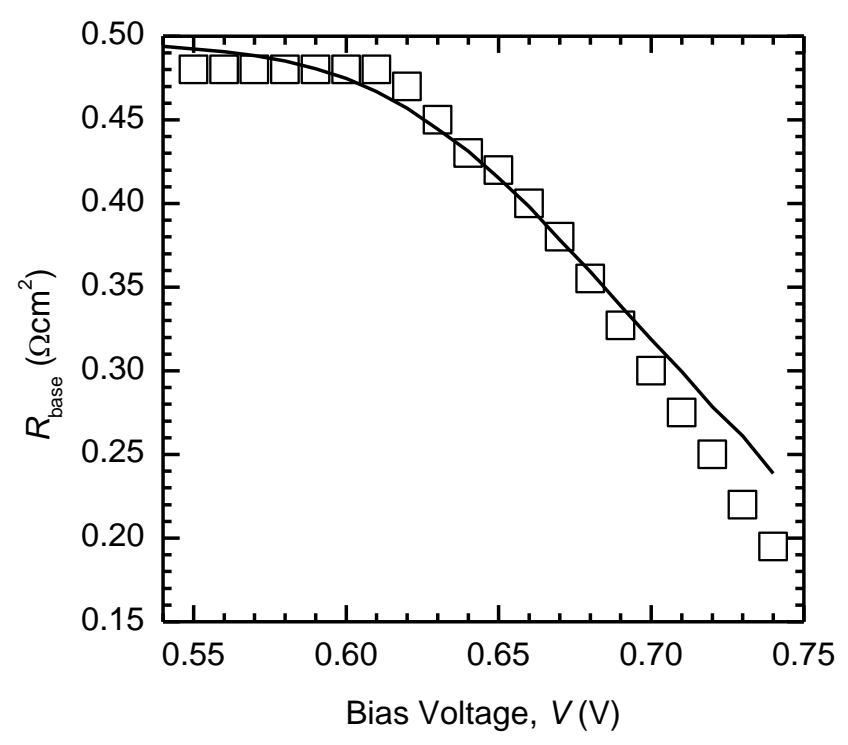

Fig.7. Experimental dependence of $R_{\text {base }}$ on bias voltage (symbols) determined from IS measurements. The analytical model (line) is able to reproduce the dependence with a contact radius of $33 \mu \mathrm{m}$ instead of $40 \mu \mathrm{m}$ determined by laser spot observation. 
Table 1. Parameter values for the theoretical curves shown in Fig. 1.

\begin{tabular}{cccccccc}
\hline \hline & $R_{0}$ & $R_{1}$ & $C_{1}$ & $L$ & $R_{2}$ & $C_{2}$ & $R_{\text {base }}$ \\
Structure & $\left(\Omega \mathrm{cm}^{2}\right)$ & $\left(\Omega \mathrm{cm}^{2}\right)$ & $\left(\mathrm{F} / \mathrm{cm}^{2}\right)$ & $(\mathrm{cm})$ & $\left(\Omega \mathrm{cm}^{2}\right)$ & $\left(\mathrm{F} / \mathrm{cm}^{2}\right)$ & $\left(\Omega \mathrm{cm}^{2}\right)$ \\
\hline PC & 0.033 & 0.510 & $12 \times 10^{-5}$ & 0.040 & - & - & 0.280 \\
PC+MIS & 0.033 & 0.510 & $12 \times 10^{-5}$ & 0.040 & 0.037 & $22 \times 10^{-9}$ & 0.280 \\
\hline \hline
\end{tabular}

